\title{
A Multivariate Lévy Process Model with Linear Correlation
}

\author{
REIICHIRO KAWAI
}

\begin{abstract}
In this paper, we develop a multivariate risk-neutral Lévy process model and discuss its applicability in the context of the volatility smile of multiple assets. Our formulation is based upon a linear combination of independent univariate Lévy processes and can easily be calibrated to a set of one-dimensional marginal distributions and a given linear correlation matrix. We derive conditions for our formulation and the associated calibration procedure to be well defined and provide some examples associated with particular Lévy processes permitting closed form characteristic function. Numerical results of the option premiums on three currencies are presented to illustrate the effectiveness of our formulation with different linear correlation structures.
\end{abstract}

Keywords: Characteristic function, implied volatility, infinitely divisible distributions, Lévy processes, linear correlation, multivariate modeling.

2000 Mathematics Subject Classification: 62H20, 60E07, 60E10, 62P05.

\section{Introduction}

Pure-jump Lévy processes and their marginal infinite divisibility have attracted considerable attention amongst practitioners and academics for the primal reason that the flexibility of their distributions, such as heavy tails and asymmetry, suits very well to various practical objects in, for example, finance, telecommunications, turbulence, to mention just a few. Particularly, in mathematical finance, there exists a vast literature on Lévy process modeling for a single asset (see, for example, Carr et al. [6], Kou [15], Madan et al.[17], Prause [22], Rydberg [23], Schoutens and Teugels [25]). In the recent structured products market, it has become quite usual that the coupons are determined by more than a single index, for example, two FX rates of USDJPY and AUDJPY. However, once the pricing model for multi-asset exotics is required to get beyond the Black-Scholes framework,

Published in Quantitative Finance (2009) 9(5) 597-606.

Email address: reiichiro_kawai@ybb.ne.jp. Postal address: Center for the Study of Finance and Insurance, Osaka University, Toyonaka, 560-8531, Japan. TEL:+81-6-6850-6279. FAX:+81-6-6850-6092. 
there is still no market consensus on how to model both volatility smiles and an intended correlation structure. In the literature, there has recently been an increasing interest in the multivariate Lévy process modeling. For example, the Lévy copula models of Kallsen and Tankov [13] and Tankov [26] completely characterize the law of a multivariate Lévy process, and are applied in pricing basket options, while Luciano and Schoutens [16] and Moosbrucker [19] propose to produce some dependence among components in the variance gamma process framework by setting a (fully or partially) common time-changing stochastic process for every component. A general construction of multi-factor Lévy models from Lévy models on rays is studied in Boyarchenko and Levendorskii [4]. Among other examples of the multivariate model related to Lévy processes and infinitely divisible distributions are a multidimensional structure model of discount factors of Bakshi [1], while various credit derivatives models in Baxter [2], Hull and White [11], O'Kane and Schloegl [20] and Kalemanova et al.[12].

In this paper, we propose a multivariate risk-neutral Lévy process model, which is based on a linear combination of independent univariate Lévy processes, and which can easily be calibrated to a set of one-dimensional marginal distributions and a given linear correlation matrix. Our formulation has three contributing features. First, applying Lévy processes permitting a parametric form of the characteristic function, we can easily derive the conditions for both our formulation and the Carr-Madan method [8] to be well defined (Proposition 3.2). Second, our model is built so as to precisely satisfy a given linear correlation matrix. Regardless of the increasing interest in the tail dependence in the extreme value theory, from a viewpoint of financial practitioners, it is usually more important to make sure to attain an intended linear correlation. Although, as claimed in the literature, the linear correlation may be too simple, it is in fact complicated enough in the sense that its change is unhedgable even in the Black-Scholes framework. Third and perhaps most importantly, compared to existing models, our formulation provides a considerably easier way to simulate the resultant multivariate Lévy process, as a linear combination of independent univariate Lévy processes.

The rest of this paper is organized as follows. Section 2 gives some general notations and motivates our model development. Section 3 formulates our model and derives conditions for the entire calibration procedure to be well defined. We also provide some explicit examples associated with particular Lévy processes permitting closed form characteristic function. Section 4 presents numerical results on the option premiums of three currencies, USDJPY, EURJPY and AUDJPY, with different linear correlation structures and also considers the extreme value dependence. Finally, Section 5 concludes. 


\section{Preliminaries}

Let us begin with some notations and definitions which will be used in what follows. $\mathbb{R}^{d}$ is the $d$-dimensional Euclidean space with the norm $\|\cdot\|, \mathbb{R}_{0}^{d}:=\mathbb{R}^{d} \backslash\{0\}$, and $\mathscr{B}\left(\mathbb{R}_{0}^{d}\right)$ is the Borel $\sigma$-field of $\mathbb{R}_{0}^{d} . \mathscr{L}(X)$ denotes the law of the random vector $X$, while $\stackrel{\mathscr{L}}{=}$ is the equality in law. We denote by $\mathbb{G} \in \mathbb{R}^{d \times d}$ the linear correlation matrix for a $d$-dimensional random vector in the form of

$$
\mathbb{G}:=\left(\begin{array}{ccccc}
1 & \rho_{1,2} & \rho_{1,3} & \cdots & \rho_{1, d} \\
\rho_{2,1} & 1 & \rho_{2,3} & \cdots & \rho_{2, d} \\
\rho_{3,1} & \rho_{3,2} & 1 & \cdots & \rho_{3, d} \\
\vdots & \vdots & \vdots & \ddots & \vdots \\
\rho_{d, 1} & \rho_{d, 2} & \rho_{d, 3} & \cdots & 1
\end{array}\right),
$$

which is symmetric positive definite and where for each $i, j,\left|\rho_{i, j}\right| \leq 1$. A stochastic process $\left\{X_{t}: t \geq 0\right\}$ in $\mathbb{R}^{d}$ is called a Lévy process if it has stationary and independent increments, if it is stochastically continuous, and if $X_{0}=0$ almost surely. It is well known that the marginal distributions of a Lévy process are infinitely divisible, that is, by the Lévy-Khintchine representation, their characteristic function is uniquely characterized as

$$
\mathbb{E}\left[e^{i\left\langle y, X_{t}\right\rangle}\right]=\exp \left[t\left(i\left\langle y, \gamma_{1}\right\rangle-\frac{1}{2}\langle y, A y\rangle+\int_{\mathbb{R}_{0}^{d}}\left(e^{i\langle y, z\rangle}-1-i\langle y, z\rangle \mathbb{1}_{(0,1]}(\|z\|)\right) v(d z)\right)\right],
$$

where $\gamma_{1} \in \mathbb{R}^{d}, A \in \mathbb{R}^{d \times d}$ is symmetric non-negative definite, and $v$ is a Lévy measure, that is, a $\sigma$-finite measure on $\mathbb{R}_{0}^{d}$, satisfying $\int_{\mathbb{R}_{0}^{d}}\left(\|z\|^{2} \wedge 1\right) v(d z)<+\infty$. In this paper, we will only consider Lévy processes with finite second moment. The above characteristic function can then be rewritten as

$$
\mathbb{E}\left[e^{i\left\langle y, X_{t}\right\rangle}\right]=\exp \left[t\left(i\left\langle y, \gamma_{2}\right\rangle-\frac{1}{2}\langle y, A y\rangle+\int_{\mathbb{R}_{0}^{d}}\left(e^{i\langle y, z\rangle}-1-i\langle y, z\rangle\right) v(d z)\right)\right],
$$

where the Lévy measure $v$ is now in $L^{2}\left(\mathbb{R}_{0}^{d}\right)$, that is, $\int_{\mathbb{R}_{0}^{d}}\|z\|^{2} v(d z)<+\infty$. It also holds that every infinitely divisible distribution with finite second moment is uniquely characterized by the three parameters $\left(\gamma_{2}, A, v\right)$ in the representation (2.1). We denote by $\varphi$ the distinguished logarithm of the characteristic function (2.1), that is, $\varphi(y):=\ln \mathbb{E}\left[e^{i\left\langle y, X_{1}\right\rangle}\right]$. For convenience, we will write $\varphi_{X}$ for the distinguished logarithm for the random vector $X$.

Consider a non-trivial $d$-dimensional random vector

$$
Z:=\left(Z_{1}, \cdots, Z_{d}\right)^{\prime}
$$

whose law is known componentwise and such that $\mathbb{E}\left[\|Z\|^{2}\right]<+\infty$. Define also the following notations,

(i) the mean vector $\mu:=\left(\mu_{1}, \ldots, \mu_{d}\right)^{\prime}:=\mathbb{E}[Z]$, 
(ii) the standard-deviation vector $\sigma:=\left(\sigma_{1}, \ldots, \sigma_{d}\right)^{\prime}:=\left[\mathbb{E}\left[(Z-\mu)^{2}\right]\right]^{1 / 2}$,

The main scope of our model is to ease the calibration procedure in finding a set of $(C, X)$, where $C \in \mathbb{R}^{d \times d}$ and $X:=\left(X_{1}, \ldots, X_{d}\right)^{\prime}$ is an infinitely divisible random vector in $\mathbb{R}^{d}$ with independent components in the following sense;

(i) the law of $C X$ approximates that of $Z$ componentwise, and

(ii) the correlation matrix of $C X$ is approximately or precisely $\mathbb{G}$, which we give as an exogenous factor.

To motivate our discussion, let us begin with the simplest yet very illustrative example. Suppose the random vector $Z$ is purely-Gaussian. Let $X$ be a $d$-dimensional standard normal random vector (with independent components) and let $C$ be the lower-triangular matrix obtained via the Cholesky decomposition of the variance-covariance matrix $\operatorname{diag}(\sigma) \mathbb{G} \operatorname{diag}(\sigma)$. It is then evident that the law of $C X+\mu$ is identical to (is not only an approximation of) that of $Z$, since the normal random vector is characterized by the mean vector and the variance-covariance matrix. In most cases, the matrix $C$ is taken the lower-triangular Cholesky matrix, since then $C X$ does not require full matrix multiplication due to the lower-triangular structure of $C$. Note however that $C$ can also be any matrix satisfying $C C^{\prime}=A$. For example, it can be a matrix obtained via the singular value decomposition.

Let us next apply the same approach to the case when $Z$ is infinitely divisible without Gaussian component. For simplicity, we assume that the random vector $Z$ is in $\mathbb{R}^{2}$ and $C \in \mathbb{R}^{2 \times 2}$ is the lowertriangular Cholesky matrix of $\operatorname{diag}(\sigma) \mathbb{G} \operatorname{diag}(\sigma)$, whose $(i, j)$-element is denoted by $c_{i, j}$. For each component $k$, we denote by $v_{k}$ the Lévy measure of $Z_{k}$, that is,

$$
\mathbb{E}\left[e^{i y z_{k}}\right]=\exp \left[i y \mu_{k}+\int_{\mathbb{R}_{0}}\left(e^{i y z}-1-i y z\right) v_{k}(d z)\right]
$$

We wish to find a random vector $X$ which achieves

$$
\begin{aligned}
& \frac{Z_{1}-\mu_{1}}{\sigma_{1}} \stackrel{\mathscr{L}}{=} c_{1,1} X_{1}, \\
& \frac{Z_{2}-\mu_{2}}{\sigma_{2}} \stackrel{\mathscr{L}}{=} c_{2,1} X_{1}+c_{2,2} X_{2} .
\end{aligned}
$$

The law of the first component can be easily recovered by setting the characteristic function

$$
\mathbb{E}\left[e^{i y X_{1}}\right] \leftarrow \psi_{1}(y):=\mathbb{E}\left[\exp \left(i y \frac{Z_{1}-\mu_{1}}{c_{1,1} \sigma_{1}}\right)\right] .
$$

Let $T_{r}$ be a transformation defined by $\left(T_{r} v\right)(B):=v\left(r^{-1} B\right)$ for $B \in \mathscr{B}\left(\mathbb{R}_{0}^{d}\right)$. For the second com- 
ponent, using the independence between $X_{1}$ and $X_{2}$, we are to set

$$
\begin{aligned}
\mathbb{E}\left[e^{i y X_{2}}\right] \leftarrow \psi_{2}(y):=\mathbb{E}\left[\exp \left(i y \frac{Z_{2}-\mu_{2}}{c_{2,2} \sigma_{2}}\right)\right] / \mathbb{E}\left[\exp \left(i y \frac{c_{2,1}}{c_{2,2}} \frac{Z_{1}-\mu_{1}}{c_{1,1} \sigma_{1}}\right)\right] \\
=\exp \left[i y\left(\frac{c_{2,1}}{c_{2,2}} \frac{\mu_{1}}{c_{1,1} \sigma_{1}}-\frac{\mu_{2}}{c_{2,2} \sigma_{2}}\right)\right. \\
\left.\quad+\int_{\mathbb{R}_{0}}\left(e^{i y z}-1-i y z\right)\left(T_{\frac{1}{c_{2,2} \sigma_{2}}} v_{2}-T_{\frac{c_{2,1}}{c_{2,2}} \frac{1}{c_{1,1} \sigma_{1}}} v_{1}\right)(d z)\right],
\end{aligned}
$$

where the division in (2.3) is well defined since the characteristic function of infinitely divisible distributions is necessarily nonzero. A crucial drawback appearing here is that the above measure

$$
T_{\frac{1}{c_{2,2} \sigma_{2}}} v_{2}-T_{\frac{c_{2,1}}{c_{2,2}} \frac{1}{c_{1,1} \sigma_{1}}} v_{1}
$$

might not be a Lévy measure. More precisely, this is clearly in $L^{2}\left(\mathbb{R}_{0}^{d}\right)$, while it might not be a non-negative measure anymore. If that is the case, then $\psi_{2}$ is not necessarily a characteristic function of any distribution.

Example 2.1. The Meixner distribution of [25] is infinitely divisible and its Lévy measure is characterized by 3 parameters $(a, b, d)$ in the form of

$$
v(d z)=d \frac{\exp (b z / a)}{z \sinh (\pi z / a)} d z, \quad z \in \mathbb{R}_{0}
$$

where $a>0, b \in(-\pi, \pi)$, and $d>0$. Now, let $Z_{1}$ and $Z_{2}$ be identically distributed Meixner random variables whose common Lévy measure is set with the parameters $(a, b)=(1,-2)$ and $d=2(\cos (b / 2) / a)^{2}$. This formulation guarantees $\mathbb{E}\left[Z_{1}\right]=0$ and $\operatorname{Var}\left(Z_{1}\right)=1$. Following the the aforementioned procedure, we find independent random variables $X_{1}$ and $X_{2}$, for example, with the correlation matrix

$$
\mathbb{G}=\left(\begin{array}{cc}
1 & -0.6 \\
-0.6 & 1
\end{array}\right) .
$$

A numerical experiment shows that the measure (2.4) falls below zero approximately for $z>0.27$. Such a measure certainly cannot be a Lévy measure.

\section{Model formulation}

Let us now proceed to our model formulation. To keep the full generality of the discussion, we drop the assumption of the lower-triangular structure of the matrix $C \in \mathbb{R}^{d \times d}$.

Let $\left\{X_{t}: t \geq 0\right\}$ be a Lévy process in $\mathbb{R}^{d}$ without Gaussian component and with independent components. Moreover, we mean by $\left\{X_{t, k}: t \geq 0\right\}$ the $k$-th component of $\left\{X_{t}: t \geq 0\right\}$, and denote 
by $v_{k}$ its Lévy measure on $\mathbb{R}_{0}$. Due to the component independence, the Lévy process can be characterized componentwise in full. We denote by $\varphi_{k}$ the distinguished logarithm of the $k$-th component, that is, $e^{\varphi_{k}(y)}:=\mathbb{E}\left[e^{i y X_{1, k}}\right]$. Let us then put the following assumption in force,

$$
\operatorname{Var}\left(X_{t, 1}\right)=\cdots=\operatorname{Var}\left(X_{t, d}\right)=t \xi^{2}
$$

for some $\xi>0$.

Next, let $\left\{Z_{t}: t \geq 0\right\}$ be a stochastic process in $\mathbb{R}^{d}$, with its $k$-th component written as $\left\{Z_{t, k}\right.$ : $t \geq 0\}$ and defined by

$$
Z_{t, k}:=\sum_{l=1}^{d}\left(c_{k, l} X_{t, l}-t \varphi_{l}\left(-i c_{k, l}\right)\right), \quad t \geq 0,
$$

provided that for all $k$ and $l$,

$$
\left|\varphi_{l}\left(-i c_{k, l}\right)\right|<+\infty
$$

By the assumption (3.1), this formulation guarantees that the given linear correlation matrix $\mathbb{G}$ is attained, that is, for each $t \geq 0$ and for $k_{1}, k_{2}$,

$$
\begin{aligned}
\operatorname{Corr}\left(Z_{t, k_{1}}, Z_{t, k_{2}}\right) & =\frac{\operatorname{Cov}\left(Z_{t, k_{1}}, Z_{t, k_{2}}\right)}{\sqrt{\operatorname{Var}\left(Z_{t, k_{1}}\right) \operatorname{Var}\left(Z_{t, k_{2}}\right)}} \\
& =\sum_{l=1}^{d} c_{k_{1}, l} c_{k_{2}, l} \\
& =\rho_{k_{1}, k_{2}} .
\end{aligned}
$$

Remark 3.1. The independence of the components implies that the Lévy measure $v$ on $\mathbb{R}_{0}^{d}$ of $\left\{X_{t}: t \geq 0\right\}$ is supported on the axes of $\mathbb{R}^{d}$ and can be written as

$$
v\left(d z_{1}, \ldots, d z_{d}\right)=\sum_{k=1}^{d} \delta_{0}\left(d z_{1}\right) \cdots \delta_{0}\left(d z_{k-1}\right) v_{k}\left(d z_{k}\right) \delta_{0}\left(d z_{k+1}\right) \cdots \delta_{0}\left(d z_{d}\right)
$$

where $\delta_{0}$ is the Dirac delta measure at zero. After the linear transformation by the matrix $C$, the Lévy measure on $\mathbb{R}_{0}^{d}$ of $\left\{Z_{t}: t \geq 0\right\}$ is given by $\left(T_{C} v\right)(B):=v\left(C^{-1} B\right)$ for $B \in \mathscr{B}\left(\mathbb{R}_{0}^{d}\right)$. Notice that it is supported on $d$ lines in $\mathbb{R}^{d},\left\{a\left(c_{1, k}, \cdots, c_{d, k}\right)^{\prime}: a \in \mathbb{R}\right\}_{k=1, \ldots, d}$. In particular, if the matrix $C$ is the one obtained via the singular value decomposition, its support is the union of all the eigenspaces of the correlation matrix $\mathbb{G}$.

Now, let $r$ be a non-negative (constant) risk free rate and let $\left\{S_{t}: t \geq 0\right\}$ be a stochastic process in $\mathbb{R}^{d}$, again with its $k$-th component written as $\left\{S_{t, k}: t \geq 0\right\}$ and defined by

$$
S_{t, k}:=e^{r t} S_{0, k} \exp \left(Z_{t, k}\right), \quad t \geq 0,
$$

where $S_{0, k}$ is a positive constant. It is immediate that for each $k$, the discounted process of $\left\{S_{t, k}: t \geq\right.$ $0\}$ is a martingale with respect to the natural filtration generated by the Lévy process $\left\{X_{t}: t \geq 0\right\}$, 
since for each $t>0$,

$$
\exp \left[\sum_{l=1}^{d}\left(c_{k, l} X_{t, l}-t \varphi_{l}\left(-i c_{k, l}\right)\right)\right]=\frac{e^{\sum_{l=1}^{d} c_{k, l} X_{t, l}}}{\mathbb{E}\left[e^{\sum_{l=1}^{d} c_{k, l} X_{t, l}}\right]},
$$

where the last equality holds by the component independence of $\left\{X_{t}: t \geq 0\right\}$.

Next, fix $T>0$ and let $\widehat{\mu}_{k}$ be the characteristic function of the logarithm of the $k$-th component of $S_{T}$, that is,

$$
\begin{aligned}
\widehat{\mu}_{k}(y) & :=\mathbb{E}\left[e^{i y \ln S_{T, k}}\right] \\
& =e^{i y\left(r T+\ln S_{0, k}\right)} \mathbb{E}\left[e^{i y Z_{t, k}}\right] \\
& =e^{i y\left(r T+\ln S_{0, k}\right)} \prod_{l=1}^{d} \frac{e^{t \varphi_{l}\left(c_{k, l} y\right)}}{e^{i y t \varphi_{l}\left(-i c_{k, l}\right)}}
\end{aligned}
$$

When its closed form is available and when the maturity $T$ is not very small, the vanilla call option price with strike $K>0$, that is,

$$
C(K):=e^{-r T} \mathbb{E}\left[\left(S_{T, k}-K\right)_{+}\right],
$$

can be efficiently computed using the well known method of Carr and Madan [8],

$$
C(K)=\frac{e^{-r T-\alpha \ln K}}{\pi} \int_{0}^{\infty} \operatorname{Re}\left[\frac{e^{-i y \ln K} \widehat{\mu}_{k}(y-(\alpha+1) i)}{\alpha^{2}+\alpha-y^{2}+i(2 \alpha+1) y}\right] d y,
$$

where $\alpha$ is a positive constant satisfying

$$
\mathbb{E}\left[S_{T, k}^{\alpha+1}\right]<+\infty
$$

Noting that for each $k$,

$$
\begin{aligned}
\mathbb{E}\left[S_{T, k}^{\alpha+1}\right] & =\left(e^{r T} S_{0, k}\right)^{\alpha+1} \prod_{l=1}^{d} \mathbb{E}\left[e^{(\alpha+1) c_{k, l} X_{T, l}-(\alpha+1) T \varphi_{l}\left(-i c_{k, l}\right)}\right] \\
& =\left(e^{r T} S_{0, k}\right)^{\alpha+1} \prod_{l=1}^{d} \frac{e^{T \varphi_{l}\left(-i(\alpha+1) c_{k, l}\right)}}{e^{T(\alpha+1) \varphi_{l}\left(-i c_{k, l}\right)}}
\end{aligned}
$$

in order for both (3.3) and (3.6) to hold, we need to have that for every $k$ and $l$,

$$
\left|\varphi_{l}\left(-i c_{k, l}\right)\right| \vee\left|\varphi_{l}\left(-i(\alpha+1) c_{k, l}\right)\right|<+\infty
$$

The following rewrites the conditions (3.3) and (3.7) as some integrability ones of the underlying Lévy measure. 
Proposition 3.2. The condition (3.3) holds if and only if for each $l$,

$$
\int_{1}^{+\infty} e^{\max _{k}\left(c_{k, l}\right) z} v_{l}(d z)<+\infty
$$

and

$$
\int_{-\infty}^{-1} e^{\min _{k}\left(c_{k, l}\right) z} v_{l}(d z)<+\infty .
$$

Moreover, the condition (3.7) holds if and only if for each $l$,

$$
\int_{1}^{+\infty} e^{\max \left[(\alpha+1) \max _{k}\left(c_{k, l}\right), \max _{k}\left(c_{k, l}\right)\right] z} v_{l}(d z)<+\infty,
$$

and

$$
\int_{-\infty}^{-1} e^{\min \left[(\alpha+1) \min _{k}\left(c_{k, l}\right), \min _{k}\left(c_{k, l}\right)\right] z} v_{l}(d z)<+\infty .
$$

Proof. By Theorem 25.17 (ii) of Sato [24], $\varphi_{l}(-i c)$ is well defined if and only if $\int_{|z|>1} e^{c z} v_{l}(d z)<$ $+\infty$. Therefore, (3.3) holds if and only if

$$
\int_{|z|>1} e^{\max _{k}\left(c_{k, l} z\right)} v_{l}(d z)<+\infty
$$

while (3.7) holds if and only if

$$
\int_{|z|>1} e^{\max _{k}\left(c_{k, l} z \vee(\alpha+1) c_{k, l} z\right)} v_{l}(d z)<+\infty,
$$

for every $l$. With $\alpha>0$, the rest is straightforward.

Remark 3.3. In view of (3.2) and (3.4), an addition of the drift to each component $\left\{X_{t, k}: t \geq 0\right\}$ cancels out. We may thus define the distinguished logarithm at our convenience, such as

$$
\begin{gathered}
\varphi_{k}(y)=\int_{\mathbb{R}_{0}}\left(e^{i y z}-1\right) v_{k}(d z), \\
\varphi_{k}(y)=\int_{\mathbb{R}_{0}}\left(e^{i y z}-1-i y z\right) v_{k}(d z),
\end{gathered}
$$

or

$$
\varphi_{k}(y)=\int_{\mathbb{R}_{0}}\left(e^{i y z}-1-i y z \mathbb{1}_{(0, c)}(|z|)\right) v_{k}(d z),
$$

for some $c>0$, whenever those integrals are well defined.

Example 3.4. (Meixner process) Suppose that the $l$-th component $\left\{X_{t, l}: t \geq 0\right\}$ is a Meixner process with the parameter $(a, b, d)$, as described in Example 2.1. Its variance is then given in closed form

$$
\int_{\mathbb{R}_{0}} z^{2} v(d z)=\frac{d}{2}\left(\frac{a}{\cos (b / 2)}\right)^{2}
$$


while the distinguished logarithm is as simple as

$$
\varphi(y)=2 d\left[\ln \left(\cos \frac{b}{2}\right)-\ln \left(\cosh \frac{a y-i b}{2}\right)\right] .
$$

We can show that the condition (3.7) reduces to

$$
\frac{\pi-b}{a}>(\alpha+1) \max \left(0, \max _{k}\left(c_{k, l}\right)\right)
$$

and

$$
\frac{\pi+b}{a}>-(\alpha+1) \min \left(0, \min _{k}\left(c_{k, l}\right)\right) .
$$

Note that the above domain of $(a, b)$ is not quite simple for the calibration procedure, in the sense that the parameters $a$ and $b$ restrict each other.

Example 3.5. (Variance gamma process) The variance gamma process of [17] is a Lévy process obtained by evaluating Brownian motion with drift at a random time given by a gamma process;

$$
X_{t}=\theta Y_{t}+\sigma W_{Y_{t}},
$$

where $\left\{W_{t}: t \geq 0\right\}$ is a standard Brownian motion and where $\left\{Y_{t}: t \geq 0\right\}$ is a gamma process whose marginal is characterized by $\mathbb{E}\left[e^{i y Y_{t}}\right]=(1-i y \eta)^{-t / \eta}$. (See also Boyarchenko and Levendorskii [3].) Its Lévy measure is given in the form of

$$
v(d z)=\frac{\exp \left[\frac{\theta}{\sigma^{2}} z-\frac{1}{\sigma} \sqrt{\frac{2}{\eta}+\frac{\theta^{2}}{\sigma^{2}}}|z|\right]}{\eta|z|} d z, \quad z \in \mathbb{R}_{0},
$$

where $\eta>0, \theta \in \mathbb{R}$, and $\sigma>0$. This also implies that the variance gamma process can be viewed as the difference of two independent gamma processes. Its variance is given in closed form

$$
\int_{\mathbb{R}_{0}} z^{2} v(d z)=\eta \theta^{2}+\sigma^{2}
$$

while the distinguished logarithm is as simple as

$$
\varphi(y)=-\frac{1}{\eta} \ln \left(1-i \eta \theta y+\frac{\eta \sigma^{2}}{2} y^{2}\right) .
$$

We can show that the condition (3.7) holds when

$$
-\frac{\theta}{\sigma^{2}}+\frac{1}{\sigma} \sqrt{\frac{2}{\eta}+\frac{\theta^{2}}{\sigma^{2}}}>(\alpha+1) \max \left(0, \max _{k}\left(c_{k, l}\right)\right),
$$

and

$$
\frac{\theta}{\sigma^{2}}+\frac{1}{\sigma} \sqrt{\frac{2}{\eta}+\frac{\theta^{2}}{\sigma^{2}}}>-(\alpha+1) \min \left(0, \min _{k}\left(c_{k, l}\right)\right) .
$$

We observe that, as in Example 3.4, the parameters $\theta, \sigma$, and $\eta$ restrict each other in a complex manner. 
Example 3.6. (CGMY process) The CGMY process proposed in [6, 7] is also a Lévy process obtained by tempering the Lévy measure of the non-Gaussian stable distribution, and its Lévy measure is given in the form of

$$
v(d z)=\left[C_{n} \frac{e^{-G|z|}}{|z|^{1+Y_{n}}} \mathbb{1}(z<0)+C_{p} \frac{e^{-M z}}{z^{1+Y_{p}}} \mathbb{1}(z>0)\right] d z, \quad z \in \mathbb{R}_{0}
$$

where $C_{n}, C_{p}, G, M>0$ and $Y_{n}, Y_{p} \in(-\infty, 2)$. Its variance is given in closed form

$$
\int_{\mathbb{R}_{0}} z^{2} v(d z)=C_{n} \frac{\Gamma\left(2-Y_{n}\right)}{G^{2-Y_{n}}}+C_{p} \frac{\Gamma\left(2-Y_{p}\right)}{M^{2-Y_{p}}},
$$

while the distinguished logarithm is as simple as

$$
\varphi(y)=C_{n} \Gamma\left(-Y_{n}\right)\left[(G+i y)^{Y_{n}}-G^{Y_{n}}\right]+C_{p} \Gamma\left(-Y_{p}\right)\left[(M-i y)^{Y_{p}}-M^{Y_{p}}\right]
$$

provided that $Y_{n} \neq 1$ and $Y_{p} \neq 1$. We can show that the condition (3.7) are

$$
G \begin{cases}\geq-(\alpha+1) \min \left(0, \min _{k}\left(c_{k, l}\right)\right), & \text { if } Y_{n} \in(0,2), \\ >-(\alpha+1) \min \left(0, \min _{k}\left(c_{k, l}\right)\right), & \text { otherwise. }\end{cases}
$$

and

$$
M \begin{cases}\geq(\alpha+1) \max \left(0, \max _{k}\left(c_{k, l}\right)\right), & \text { if } Y_{p} \in(0,2), \\ >(\alpha+1) \max \left(0, \max _{k}\left(c_{k, l}\right)\right), & \text { otherwise. }\end{cases}
$$

Unlike the case of the Meixner and the variance gamma distributions, each condition consists of a single parameter, and can thus be easily set in the calibration algorithm.

\section{Numerical illustration: Multi-Currency}

We consider the 1-year currency options on the three foreign exchanges; USDJPY, EURJPY, and AUDJPY, and model their mean-correcting forward forex dynamics by the following stochastic process

$$
F_{t}=\frac{e^{-r_{f} t}}{e^{-r_{d} t}} F_{0} \frac{e^{Z_{t}}}{\mathbb{E}\left[e^{Z_{t}}\right]},
$$

where $r_{d}$ and $r_{f}$ denote the continuously-compounded domestic and foreign risk-free rates, respectively. In our case, "domestic" means "JPY", while "foreign" corresponds to either one of "USD", "EUR", or "AUD". We assume for simplicity that the both risk-free rates are deterministic. $F_{0}$ is the spot FX rate, while $e^{-\left(r_{f}-r_{d}\right) t} F_{0}\left(=\mathbb{E}\left[F_{t}\right]\right)$ is the so-called forward FX rate at time $t$. For notational convenience, we henceforth replace the time indices of $\left\{F_{t}: t \geq 0\right\}$ and $\left\{Z_{t}: t \geq 0\right\}$ by FX indices, that is, $F_{1}, F_{2}$ and $F_{3}$ denote USDJPY, EURJPY and AUDJPY rates at 1-year respectively, while $Z_{1}, Z_{2}$ and $Z_{3}$ are defined analogously. 


\subsection{Data description}

In general, the market quotes for currency options are available in the form of Delta-Neutral straddle implied volatility (iv ${ }_{\mathrm{DN}}$ ) and 10- and 25-delta risk reversals (RR10 and RR25), and 10- and 25-delta strangle margins (SM10 and SM25). They have the connection with implied volatilities

$$
\begin{aligned}
& \mathrm{RR} 10=\mathrm{iv}_{10 \mathrm{C}}-\mathrm{iv_{10 } \mathrm { P }}, \quad \mathrm{SM} 10=\left(\mathrm{iv}_{10 \mathrm{C}}+\mathrm{iv}_{10 \mathrm{P}}\right) / 2-\mathrm{iv}_{\mathrm{DN}}, \\
& \mathrm{RR} 25=\mathrm{iv}_{25 \mathrm{C}}-\mathrm{iv}_{25 \mathrm{P}}, \quad \mathrm{SM} 25=\left(\mathrm{iv}_{25 \mathrm{C}}+\mathrm{iv}_{25 \mathrm{P}}\right) / 2-\mathrm{iv_{ \textrm {DN } }} .
\end{aligned}
$$

Table1 gives the 1-year implied volatilities of 2006/10/25.

\begin{tabular}{|l||c|c|c|c|c|}
\hline & iv $_{10 P}$ & iv $_{25 P}$ & iv $_{\text {DN }}$ & iv $_{25 C}$ & iv $_{10 C}$ \\
\hline USDJPY & 9.44 & 8.37 & 7.70 & 7.44 & 7.66 \\
\hline EURJPY & 8.40 & 7.59 & 7.05 & 6.86 & 7.07 \\
\hline AUDJPY & 9.70 & 8.51 & 7.60 & 7.15 & 7.27 \\
\hline
\end{tabular}

Table 1: 1-year implied volatilities of $2006 / 10 / 25$ in percentage points

The strikes for each implied volatility are derived from the equations

$$
\begin{array}{cc}
0.10=\frac{K_{10 \mathrm{C}}}{\mathbb{E}[F]} \mathscr{N}\left(d\left(\mathbb{E}[F], K_{10 \mathrm{C}}, \mathrm{iv}_{10 \mathrm{C}}\right)\right), \quad 0.25=\frac{K_{25 \mathrm{C}}}{\mathbb{E}[F]} \mathscr{N}\left(d\left(\mathbb{E}[F], K_{25 \mathrm{C}}, \mathrm{iv}_{25 \mathrm{C}}\right)\right), \\
0.10=\frac{K_{0.10 \mathrm{P}}}{\mathbb{E}[F]}\left[1-\mathscr{N}\left(d\left(\mathbb{E}[F], K_{0.10 \mathrm{P}}, \mathrm{iv}_{10 \mathrm{P}}\right)\right)\right], \quad 0.25=\frac{K_{0.25 \mathrm{P}}}{\mathbb{E}[F]}\left[1-\mathscr{N}\left(d\left(\mathbb{E}[F], K_{0.25 \mathrm{P}}, \mathrm{iv}_{25 \mathrm{P}}\right)\right)\right],
\end{array}
$$

where $\mathscr{N}$ is the cumulative standard normal distribution and $d(F, K$, iv $):=\ln (F / K) /$ iv - iv $/ 2$. Finally, we have $K_{\mathrm{DN}}=\mathbb{E}[F] \exp \left(-\mathrm{iv}_{\mathrm{DN}}^{2} / 2\right)$, which implies that the the delta-neutral strike is not identical to the ATM strike, but always smaller, $K_{\mathrm{DN}}<\mathbb{E}[F]$. We give in Table 2 the strikes, together with spot FX rates, forward FX rates $\mathbb{E}[F]$, and JPYcdf, the discount factor compounding currency basis cost on the same date. With the information in Table 2 , the call premiums are computed by JPYcdf $\times \mathbb{E}\left[(F-K)_{+}\right]$and are reported in Table 3 ,

\subsection{Underlying Lévy process}

For our numerical experiments, we employ a Lévy process whose $l$-th component has a Lévy measure given by

$$
v_{l}(d z)=\frac{1}{\eta_{l}|z|}\left[e^{-\tau_{l}|z|} \mathbb{1}(z<0)+e^{-\kappa_{l} z} \mathbb{1}(z>0)\right] d z, \quad z \in \mathbb{R}_{0}
$$

where $\eta_{l}>0, \tau_{l}>0$, and $\kappa_{l}>0$. We set the characteristic function by

$$
\mathbb{E}_{\mathbb{P}}\left[e^{i y X_{t, l}}\right]:=\exp \left[t \int_{\mathbb{R}_{0}}\left(e^{i y z}-1\right) v_{l}(d z)\right] .
$$




\begin{tabular}{|l||c|c|c|c|c|c|c|c|}
\hline & $K_{10 \mathrm{P}}$ & $K_{25 \mathrm{P}}$ & $K_{\mathrm{DN}}$ & $K_{25 \mathrm{C}}$ & $K_{10 C}$ & Spot & $\mathbb{E}[F]$ & JPYcdf \\
\hline USDJPY & 100.91 & 107.48 & 113.39 & 119.63 & 125.70 & 119.25 & 113.73 & 0.9931 \\
\hline EURJPY & 130.51 & 137.93 & 144.82 & 152.11 & 159.22 & 149.85 & 145.18 & 0.9933 \\
\hline AUDJPY & 76.01 & 80.88 & 85.41 & 89.93 & 94.19 & 90.77 & 85.66 & 0.9936 \\
\hline
\end{tabular}

Table 2: Strikes, spot FX, forward FX, and discount factor

\begin{tabular}{|l||c|c|c|c|c|}
\hline & $K_{10 \mathrm{P}}$ & $K_{25 \mathrm{P}}$ & $K_{\mathrm{DN}}$ & $K_{25 \mathrm{C}}$ & $K_{10 \mathrm{C}}$ \\
\hline USDJPY & 13.22 & 7.575 & 3.635 & 1.274 & 0.408 \\
\hline EURJPY & 15.13 & 8.791 & 4.231 & 1.497 & 0.480 \\
\hline AUDJPY & 9.996 & 5.798 & 2.702 & 0.921 & 0.292 \\
\hline
\end{tabular}

Table 3: Call premiums

From its gamma structure, it immediately follows that

$$
\begin{aligned}
e^{\varphi_{X_{t, l}}(y)} & =\left(1+\frac{i y}{\tau_{l}}\right)^{-t / \eta_{l}}\left(1-\frac{i y}{\kappa_{l}}\right)^{-t / \eta_{l}} \\
& =\left(1+i y\left(\frac{1}{\tau_{l}}-\frac{1}{\kappa_{l}}\right)\right)^{-t / \eta_{l}}
\end{aligned}
$$

and

$$
\operatorname{Var}\left(X_{t, l}\right)=\frac{t}{\eta_{l}}\left(\frac{1}{\tau_{l}^{2}}+\frac{1}{\kappa_{l}^{2}}\right)
$$

As can be seen, our Lévy process is very akin to the variance gamma process, and in fact it can even recover the variance gamma process by setting

$$
\tau=\frac{2}{\eta}\left(\sqrt{\theta^{2}+\frac{\sigma^{2}}{\eta}}-\theta\right)^{-1}
$$

and

$$
\kappa=\frac{2}{\eta}\left(\sqrt{\theta^{2}+\frac{2 \sigma^{2}}{\eta}}+\theta\right)^{-1},
$$

where the parameters $(\theta, \sigma)$ are as defined in Example 3.5. Here, by unfastening the above dependence between $\tau$ and $\kappa$, we do not only make the Lévy measure more flexible, but also simplify the conditions for (3.7), compared to those derived in Example 3.5, as simple as

$$
\left\{\begin{array}{l}
\tau_{l}>-(\alpha+1) \min \left(0, \min _{k}\left(c_{k, l}\right)\right), \\
\kappa_{l}>(\alpha+1) \max \left(0, \max _{k}\left(c_{k, l}\right)\right),
\end{array}\right.
$$


that is, each condition consists of a single parameter. Yet, our Lévy process enjoys the nice features of the variance gamma process; (i) its characteristic function is given in closed form, and (ii) it can be viewed as the difference of two independent gamma process. One thing our Lévy process does not inherit from the variance gamma process is the property of "time-changed Brownian motion" of (3.8), since the parameters $\tau$ and $\kappa$ are no longer dependent in the way of (4.3) and (4.4). From practical point of view, however, this property is not very important as long as our Lévy process can still be simulated by two independent gamma processes.

For the condition (3.1) to be satisfied, with the help of the closed form variance (4.2), we fix the parameter $\eta$ as for each $l$,

$$
\eta_{l}=\frac{1}{\xi^{2}}\left(\frac{1}{\tau_{l}^{2}}+\frac{1}{\kappa_{l}^{2}}\right) .
$$

Hence, in the calibration procedure, we will only control the rest two parameters $\left(\tau_{l}, \kappa_{l}\right)$ for the $l$-th component. Notice that under the condition (4.6), the realized linear correlation matrix of the three FXs is supposed to be precisely $\mathbb{G}$, whatever the calibration result is.

\subsection{Calibration with given correlation}

We test for the following correlation structures;

$$
\begin{gathered}
\mathbb{G}_{1}:=\left[\begin{array}{rrr}
1 & 0.25 & 0.50 \\
0.25 & 1 & 0.60 \\
0.50 & 0.60 & 1
\end{array}\right], \quad \mathbb{G}_{2}:=\left[\begin{array}{rrr}
1 & 0.80 & 0.75 \\
0.80 & 1 & 0.85 \\
0.75 & 0.85 & 1
\end{array}\right], \\
\mathbb{G}_{3}:=\left[\begin{array}{rrr}
1 & 0.65 & 0.75 \\
0.65 & 1 & 0.20 \\
0.75 & 0.20 & 1
\end{array}\right], \quad \mathbb{G}_{4}:=\left[\begin{array}{rrr}
1 & -0.25 & 0.50 \\
-0.25 & 1 & 0.60 \\
0.50 & 0.60 & 1
\end{array}\right],
\end{gathered}
$$

where $\mathbb{G}_{1}, \mathbb{G}_{2}$ and $\mathbb{G}_{3}$ are approximate historical correlations among the three FXs (USDJPY, EURJPY, AUDJPY from the first component to the third) for 200days (2006/1/16 - 2006/10/25), 100days (2006/6/5 - 2006/10/25), and 25days (2006/9/19 - 2006/10/25), respectively. We also prepare $\mathbb{G}_{4}$, which is an artificial modification of $\mathbb{G}_{1}$, to test some negative correlation. Here, we obtain the matrix $C$ such that $C C^{\prime}=\mathbb{G}$ via the singular value decomposition, and they are respectively

$$
\begin{aligned}
& C_{1}:=\left[\begin{array}{rrr}
0.2084 & 0.6782 & 0.7047 \\
0.3121 & -0.5398 & 0.7818 \\
-0.4352 & -0.0623 & 0.8982
\end{array}\right], \quad C_{2}:=\left[\begin{array}{rrr}
-0.0830 & 0.4034 & 0.9113 \\
0.2948 & -0.1024 & 0.9501 \\
-0.2194 & -0.2902 & 0.9315
\end{array}\right], \\
& C_{3}:=\left[\begin{array}{rrr}
-0.2355 & 0.0279 & 0.9715 \\
0.1332 & -0.6801 & 0.7209 \\
0.1669 & 0.5821 & 0.7958
\end{array}\right], \quad C_{4}:=\left[\begin{array}{rrr}
-0.1488 & 0.8707 & 0.4686 \\
-0.1651 & -0.6930 & 0.7018 \\
0.1897 & 0.0800 & 0.9786
\end{array}\right] .
\end{aligned}
$$


We perform the calibration via the Nelder-Mead direct search method to minimize the difference between market premiums (market-pr) and model premiums (model-pr) in the sense of the root mean square error (rmse) defined by

$$
(\text { rmse }):=\left[\frac{1}{\#(\text { pr })} \sum[(\text { market-pr })-(\text { model-pr })]^{2}\right]^{1 / 2} .
$$

In our example, $\#(\mathrm{pr})=15$ (that is, 5 premiums for each of 3 currencies) and the calibration is a minimization problem with 6 parameters $\left(\tau_{1}, \kappa_{1}, \ldots, \tau_{3}, \kappa_{3}\right)$, due to the common variance condition (3.1), where those parameters can take values in the domain (4.5). During the calibration procedure, we compute the model premiums using the Carr-Madan method (3.5).

It is well known that the calibration of infinitely divisible marginals is an ill-posed inverse problem (see for example, Cont and Tankov [9]), and so local minima are always a problem. We thus perform the minimization problem many times, with different common variance $\xi^{2}$ in (3.1), and from different randomly chosen starting points. The calibration results in Table 4 are the best one on each case. As can be seen in Figure1, our formulation is capable of fitting market premiums very well with different linear correlation structures.

\begin{tabular}{|c|c|c|c|c|c|c|c|}
\hline & $\tau$ & $\kappa$ & $\eta$ & & $\tau$ & $\kappa$ & $\eta$ \\
\hline$X_{1}$ & 9.775 & 9.415 & 3.866 & $X_{1}$ & 6.962 & 6.685 & 7.646 \\
\hline$X_{2}$ & 16.06 & 15.12 & 1.467 & $X_{2}$ & 9.684 & 9.074 & 4.055 \\
\hline$X_{3}$ & 18.38 & 19.96 & .9723 & $X_{3}$ & 18.49 & 21.10 & .9190 \\
\hline \multicolumn{4}{|c|}{$\mathbb{G}_{1}: \xi=0.075$} & \multicolumn{4}{|c|}{$\mathbb{G}_{2}: \xi=0.075$} \\
\hline & $\tau$ & $\kappa$ & $\eta$ & & $\tau$ & $\kappa$ & $\eta$ \\
\hline$X_{1}$ & 6.189 & 6.380 & 9.008 & $X_{1}$ & 5.143 & 5.029 & 13.75 \\
\hline$X_{2}$ & 17.44 & 16.37 & 1.248 & $X_{2}$ & 20.65 & 22.26 & .7757 \\
\hline$X_{3}$ & 22.37 & 24.81 & .6442 & $X_{3}$ & 35.57 & 46.00 & .2245 \\
\hline \multicolumn{4}{|c|}{$\mathbb{G}_{3}: \xi=0.075$} & \multicolumn{4}{|c|}{$\mathbb{G}_{4}: \xi=0.075$} \\
\hline
\end{tabular}

Table 4: Calibration results

Scatter plots of 500 points for currency pairs are given in Figure 2, We observe that, compared to the ordinary Gaussian framework, our Lévy process model can express a dependence in deeper negative values. These realizations can easily be generated based on a linear combination of three random variables $X_{1}, X_{2}$ and $X_{3}$. Particularly, thanks to the gamma structure of the underlying Lévy measure (4.1), each component $X_{k}$ is simulated as the difference of independent gamma random variables, of which sample generation routine is devised in many standard mathematical and statistical softwares. This simplicity in implementation should be a great advantage, 


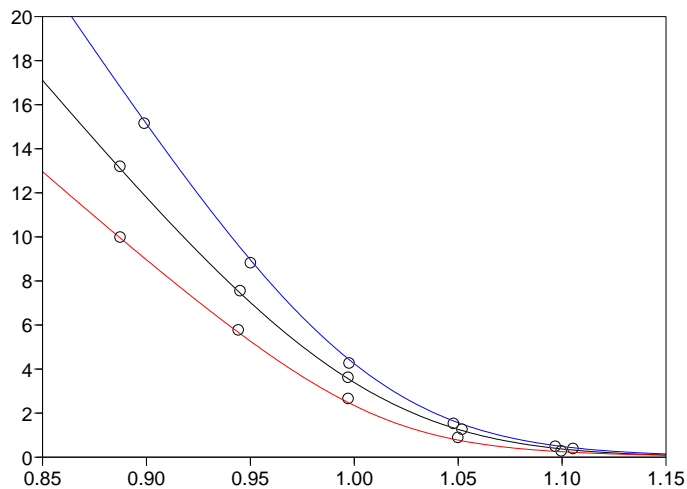

$\mathbb{G}_{1}:($ rmse $)=5.82 \mathrm{e}-2$

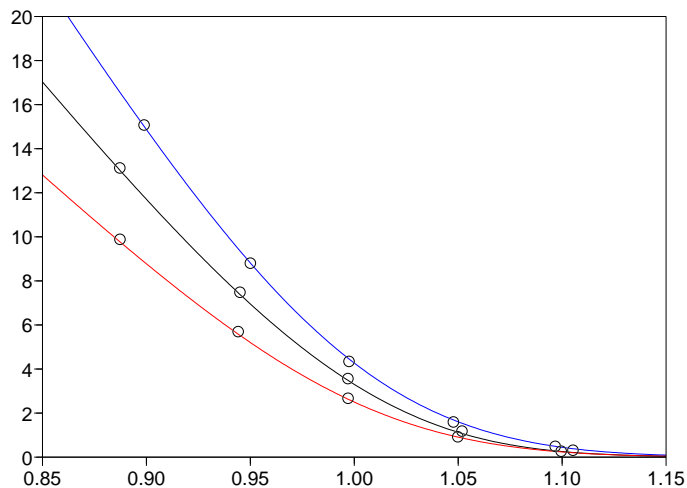

$\mathbb{G}_{3}:($ rmse $)=7.77 \mathrm{e}-2$

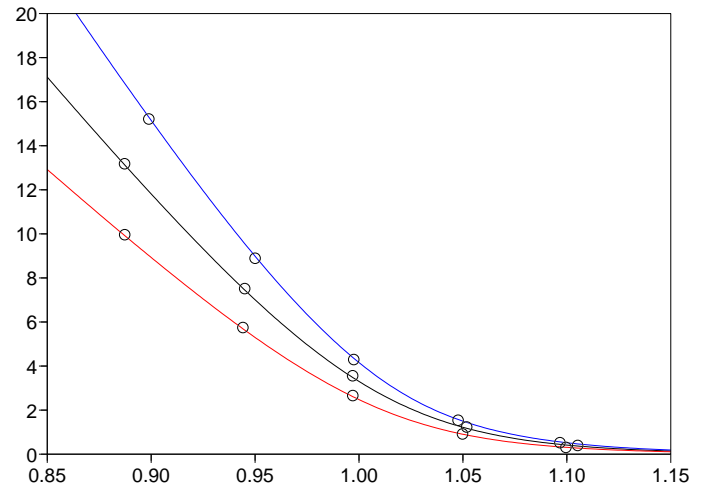

$\mathbb{G}_{2}:(\mathrm{rmse})=5.12 \mathrm{e}-2$

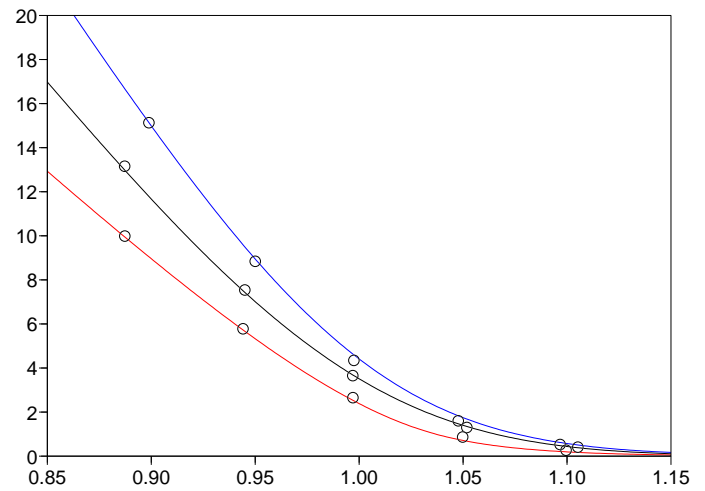

$\mathbb{G}_{4}:(\mathrm{rmse})=7.56 \mathrm{e}-2$

Figure 1: Market premiums ("o") and model premiums ("-_"). The $x$-axis indicates the moneyness of strikes, $K / \mathbb{E}[F]$. Recall that $K_{D N}<\mathbb{E}[F]$.

compared to existing multivariate Lévy process models. For example, the stochastic volatility formulation of [16] is based on a time-changed Brownian motion, and usually requires an Euler-type discretization of sample paths for simulation, while the Lévy copula model [26] resorts to the series representation of Lévy processes, which is well known to be computationally very expensive for most simulation use.

\subsection{Extreme value dependence}

Our formulation is the one to approximate a set of one-dimensional marginal distributions with a given linear correlation structure, while not modeled so as to have control over the extreme value dependence. It is worthwhile to check how our multivariate model behaves in the extreme values. 


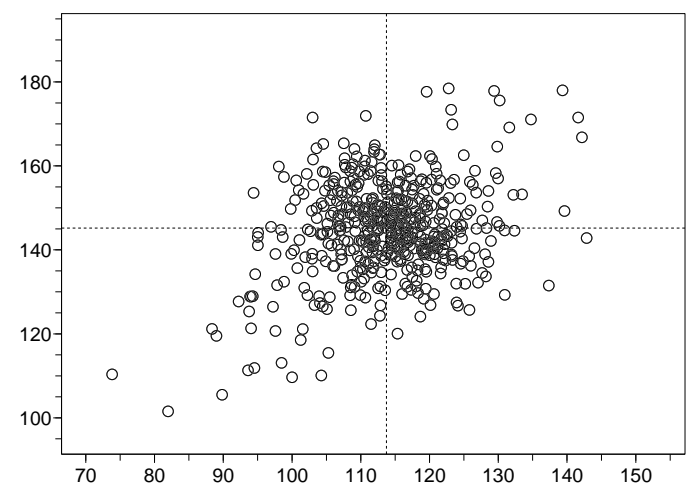

USDJPY-EURJPY $(\rho=0.25)$

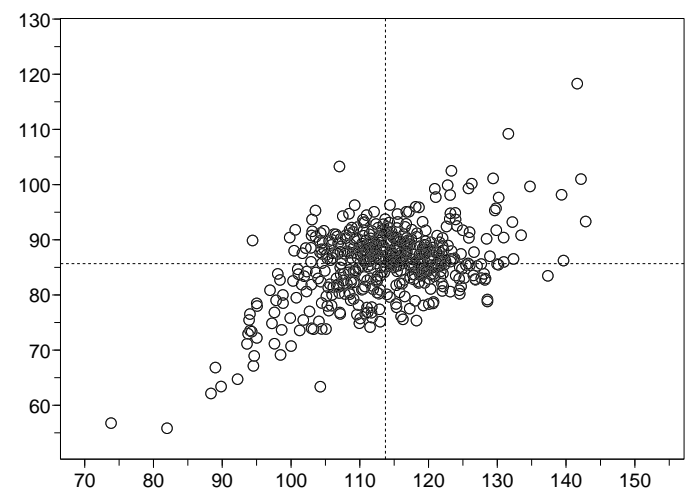

USDJPY-AUDJPY $(\rho=0.50)$

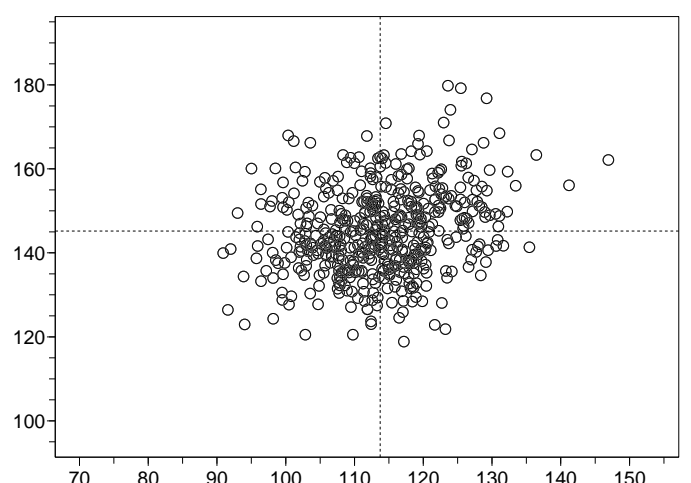

USDJPY-EURJPY (Gaussian, $\rho=0.25$ )

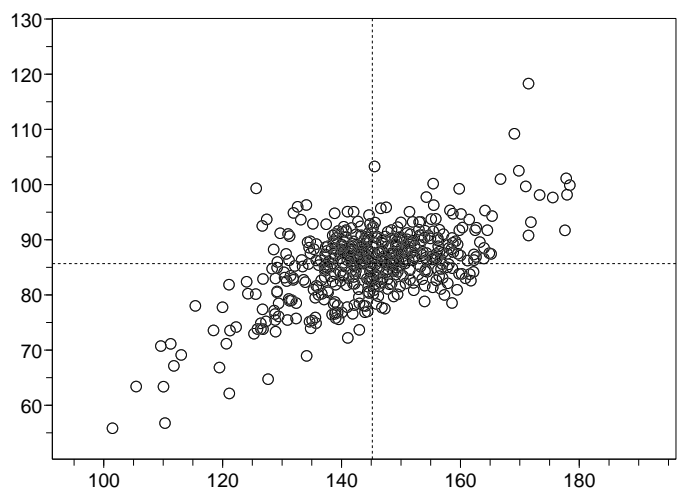

EURJPY-AUDJPY $(\rho=0.60)$

Figure 2: Scatter plots of 500 points of each currency-pair, with the correlation matrix $\mathbb{G}_{1}$. Each dotted-line indicates a corresponding forward $\mathrm{FX}$ rate $\mathbb{E}[F]$.

To this end, for a fixed $T$, and for each $k$, let $F_{k}^{-1}(u)$ be the inverse cumulative distribution function of $Z_{T, k}$, that is, $F_{k}^{-1}(u):=\inf \left\{v \in \mathbb{R}: \mathbb{P}\left(Z_{T, k} \leq v\right)>u\right\}$. Then, define the measures of the extreme value dependence,

$$
\begin{aligned}
\lambda_{L L}(u) & :=\mathbb{P}\left[Z_{T, k_{1}} \leq F_{k_{1}}^{-1}(u) \mid Z_{T, k_{2}} \leq F_{k_{2}}^{-1}(u)\right], \\
\lambda_{U U}(u) & :=\mathbb{P}\left[Z_{T, k_{1}}>F_{k_{1}}^{-1}(1-u) \mid Z_{T, k_{2}}>F_{k_{2}}^{-1}(1-u)\right], \\
\lambda_{L U}(u) & :=\mathbb{P}\left[Z_{T, k_{1}} \leq F_{k_{1}}^{-1}(u) \mid Z_{T, k_{2}}>F_{k_{2}}^{-1}(1-u)\right], \\
\lambda_{U L}(u) & :=\mathbb{P}\left[Z_{T, k_{1}}>F_{k_{1}}^{-1}(1-u) \mid Z_{T, k_{2}} \leq F_{k_{2}}^{-1}(u)\right] .
\end{aligned}
$$

We perform Monte Carlo simulation to estimate the above quantities based on $1 \mathrm{e}+5$ independent replications, and report them in Table 5. We observe quite direct results of a greater extreme 


\begin{tabular}{c||ccc|ccc|ccc|ccc}
\multicolumn{1}{c||}{} & \multicolumn{3}{|c|}{$\mathbb{G}_{1}$} & \multicolumn{3}{|c|}{$\mathbb{G}_{2}$} & \multicolumn{3}{|c}{$\mathbb{G}_{3}$} & \multicolumn{3}{|c}{$\mathbb{G}_{4}$} \\
\hline \hline$\left(k_{1}, k_{2}\right)$ & $(1,2)$ & $(2,3)$ & $(3,1)$ & $(1,2)$ & $(2,3)$ & $(3,1)$ & $(1,2)$ & $(2,3)$ & $(3,1)$ & $(1,2)$ & $(2,3)$ & $(3,1)$ \\
$\rho$ & 0.25 & 0.60 & 0.50 & 0.80 & 0.85 & 0.75 & 0.65 & 0.20 & 0.75 & -0.25 & 0.60 & 0.50 \\
\hline$\lambda_{L L}(u)$ & .245 & .430 & .316 & .675 & .750 & .659 & .325 & .184 & .495 & .011 & .269 & .103 \\
$\lambda_{U U}(u)$ & .183 & .397 & .246 & .589 & .698 & .576 & .295 & .147 & .405 & .006 & .148 & .088 \\
$\lambda_{L U}(u)$ & .064 & .014 & .006 & .000 & .003 & .009 & .002 & .097 & .001 & .248 & .004 & .003 \\
$\lambda_{U L}(u)$ & .079 & .015 & .006 & .001 & .005 & .008 & .001 & .069 & .002 & .182 & .003 & .002
\end{tabular}

Table 5: Estimated extreme-value dependence with $u=0.01$

value dependence for a greater correlation. Our multivariate model seems to be capable also of expressing the strongly correlated downside jumps, which has often been claimed important in the economics literature. We can also see that the negative correlation induces a negative extreme value dependence.

Remark 4.1. The limiting values $\lim _{u \downarrow 0} \lambda_{L L}(u)$ and $\lim _{u \downarrow 0} \lambda_{U U}(u)$ are often called, respectively, the lower and the upper tail-dependence coefficients, and their estimation methods and applications to financial markets have been intensively studied. For details, see, for example, Caillault and Guégan [5], Frahm et al.[10], Malevergne and Sornette [18], and Poon et al.[21].

Remark 4.2. We have chosen the matrix $C$ to be the one obtained via the singular value decomposition. Nevertheless, as mentioned earlier, it could be any matrix satisfying $C C^{\prime}=\mathbb{G}$, and it is natural to be tempted to instead choose the lower-triangular Cholesky matrix. For example, for the correlation matrix $\mathbb{G}_{1}$, the matrix $C_{1}$ is then

$$
C_{1}:=\left[\begin{array}{rrr}
1 & 0 & 0 \\
0.25 & 0.9682 & 0 \\
0.50 & 0.4906 & 0.7137
\end{array}\right]
$$

Unfortunately, with this lower triangular matrix $C_{1}$, we could not get a satisfactory result via a calibration problem of 6 parameters. Meanwhile, the lower triangular structure of $C_{1}$ may reduce the simultaneous calibration to a bootstrap procedure, that is, the first component is calibrated only with $1 X_{1}$, then next the second one with $0.9682 X_{2}$ where $0.25 X_{1}$ is fixed, and so on. As can be guessed, however, as this bootstrap procedure goes for later components, the calibration quality seems to get poorer. Perhaps, this is partially because our underlying Lévy measure (4.1) of 3 parameters is not flexible enough for this purpose. Indeed, our experiment indicates that the CGMY process of 6 parameters (Example 3.6) fits all the three components very well. It is however difficult to justify that the flexibility of the Lévy measure always provides significant contributions to the calibration quality in the bootstrap procedure. Yet, another concern is that the procedure can 
certainly be done in arbitrary order in which different components are calibrated. It is not clear how much this component ordering affects the calibration quality.

\section{Concluding remarks}

In this paper, we have formulated a multivariate risk-neutral Lévy process model, which can easily be calibrated to a set of one-dimensional marginal distributions and a given linear correlation matrix. Our model is built on a linear combination of independent univariate Lévy processes so as to precisely realize the given linear correlation matrix. Applying Lévy processes permitting closed form characteristic function, our formulation provides a considerably simple calibration procedure in the Carr-Madan framework. The resultant multivariate Lévy processes can easily be generated for simulation use as a linear combination of independent univariate Lévy processes. Numerical results have indicated that our model is capable of simultaneously fitting three currency options, with different linear correlation structures, and the strongly correlated downside jumps can also be well expressed.

As future research, it would be interesting to conduct further empirical analysis on different sets of currencies and different types of financial assets, for example, a set of equity indices, and with different Lévy processes, such as the CGMY process. Moreover, an extension to the multimaturity context should certainly be pursued. This may pave a way to evaluate popular, but very intricate, financial exotics in Lévy process framework, which will be studied in Kawai [14].

\section{Acknowledgments}

Part of this work was done at Daiwa Securities SMBC Co.Ltd. The author would like to thank Tatsuya Toshida and the Financial Engineering team for their support and encouragement.

\section{References}

[1] Bakshi, G., Carr, P., Wu, L. (2007) Stochastic risk premiums, stochastic skewness in currency options, and stochastic discount factors in international economics, Journal of Financial Economics, 87, 132-156.

[2] Baxter, M. (2007) Lévy simple structural models, International Journal of Theoretical and Applied Finance, 10(4) 593-606.

[3] Boyarchenko, S.I., Levendorskii, S.Z. (2000) Option pricing for truncated Lévy processes, International Journal of Theoretical and Applied Finance, 3(3) 549-552. 
[4] Boyarchenko, S., Levendorskiï, S. (2002) Non-Gaussian Merton-Black-Scholes Theory, New Jersey-London-Singapore-Hong Kong: World Scientific.

[5] Caillault, C., Guégan, D. (2005) Empirical estimation of tail dependence using copulas: application to Asian markets, Quantitative Finance, 5, 489-501.

[6] Carr, P., Geman, H., Madan, D.B., Yor, M. (2002) The fine structure of asset returns: An empirical investigation, Journal of Business, 75, 303-325.

[7] Carr, P., Geman, H., Madan, D.B., Yor, M. (2003) Stochastic volatility for Lévy processes, Mathematical Finance, 13, 345-382.

[8] Carr, P., Madan, D.B. (1999) Option valuation using the fast Fourier transform, Journal of Computational Finance, 3, 463-520.

[9] Cont, R., Tankov, P. (2006) Retrieving Lévy processes from option prices: regularization of an ill-posed inverse problem, SIAM Journal on Control and Optimization, 45, 1-25.

[10] Frahm, G., Junker, M., Schmidt, R. (2005) Estimating the tail dependence coefficient, Insurance: Mathematics and Economics, 37, 80-100.

[11] Hull, J., White, A. (2004) Valuation of a CDO and an $n$-th to Default CDS Without Monte Carlo Simulation, Journal of Derivatives, 12(2) 8-23.

[12] Kalemanova, A., Schmid, B., Werner, R. (2007) The normal inverse Gaussian distribution for synthetic CDO pricing, Journal of Derivatives, 14(3) 80-93.

[13] Kallsen, J., Tankov, P. (2006) Characterization of dependence of multidimensional Lévy processes using Lévy copulas, Journal of Multivariate analysis, 97, 1551-1572.

[14] Kawai, R. On bootstrap calibrations with piecewise Lévy processes, Submitted for publication.

[15] Kou, S. (2002) A jump-diffusion model for option pricing, Management Science, 48, 10861101.

[16] Luciano, E., Schoutens, W. (2006) A multivariate jump-driven financial asset model, Quantitative Finance, 6, 385-402.

[17] Madan, D.B., Carr, P.P., Chang, E.C. (1998) The variance gamma process and option pricing, European Finance Review, 2, 79-105.

[18] Malevergne, Y., Sornette, D. (2005) Extreme Financial Risk: From dependence to risk management, Springer. 
[19] Moosbrucker, T. (2006) Pricing CDOs with correlated variance gamma distributions, Preprint, available at http://www.cfr-cologne.de/downloads/kolloquium

[20] O'Kane, D., Schloegl, L. (2003) An analytical portfolio credit model with tail dependence, Quantitative Credit Research, Lehman Brothers.

[21] Poon, S-H., Rochinger, M., Tawn, J. (2003) Modelling extreme-value dependence in international stock markets, Statistica Sinica, 13, 929-953.

[22] Prause, K. (1999) The Generalized Hyperbolic Model: Estimation, Financial Derivatives, and Risk Measures, Dissertation Universität Freiburg i. Br.

[23] Rydberg, T. (1997) The normal inverse Gaussian Lévy process: simulation and approximation, Communications in Statistics. Stochastic Models, 13, 887-910.

[24] Sato, K. (1999) Lévy processes and infinitely divisible distributions, Cambridge University Press.

[25] Schoutens, W., Teugels, J.L. (1998) Lévy processes, polynomials and martingales, Communications in Statistics: Stochastic Models, 14, 335-349.

[26] Tankov, P. (2005) Simulation and option pricing in Lévy copula models, Mathematical Modelling of Financial Derivatives, Eds. M. Avellaneda and R. Cont, IMA volumes in Mathematics and Applications, Springer. 